\title{
Performance Analysis of MAC Protocol for Reliable Broadcast in VANETS
}

\author{
Savita \\ ${ }^{I}$ Electronics and Communication Engineering, Lovely Professional University, India
}

\begin{abstract}
Wireless communications are becoming the dominant form of transferring information, and the most active research field. In this dissertation, we will present one of the most applicable forms of Ad-Hoc networks; the Vehicular Ad-Hoc Networks (VANETs). VANET is the technology of building a robust Ad-Hoc network between mobile vehicles and each other, besides, between mobile vehicles and roadside units.Traffic maintenance in highway using broadcasting protocol is a new theme. It is important to find a reliable broadcasting protocol that is especially designed for an optimum performance of public-safety and data travelling related applications. Using RSU and OBU, there are four novel ideas presented in this research work, namely choosing the nearest following node as the network probe node, headway-based segmentation, nonuniform segmentation and application adaptive. The integration of these ideas results in a protocol that possesses minimum latency, minimum probability of collision in the acknowledgment messages and unique robustness at different speeds and traffic volumes.
\end{abstract}

Keywords - Broadcasting protocol,DSRC, headway, MATLAB,VANET

\section{Introduction}

Efforts related to traffic management in big cities led to the promising technology of building a robust wireless mobile Ad-Hoc network between vehicles (with On-Board-Units, OBUs) and roadside units (RSUs, mounted in centralized locations such as intersections, parking lots or gas stations), referred to as a Vehicular Ad-Hoc Networks (VANET). Among the main applications of VANETs, categorized as Public/Non-Public Safety (S/NS) and Vehicle-to-Vehicle/RSU (VV/VR), are co -operative collision warning (S, VV), intersection collision warning $(S, V R)$, approaching emergency vehicle warning $(S, V V)$, work zone warning $(\mathrm{S}, \mathrm{VR})$, traffic management (NS, VV or VR), toll collection (NS, VR), and Internet services (NS, VR). Due to the high mobility of vehicles, the distribution of nodes within the network changes so very rapidly and unexpectedly that wireless links are established and broken down frequently and unpredictably, eliminating any usefulness of prior topology information. VANET operations in the absence of a fixed infrastructure force OBUs to organize network resources in a distributed way. So, broadcasting of messages in VANET environments plays a crucial rule in almost every application and represents a critical challenge that needs novel solutions based on the unique characteristics of VANETs. The target is to optimally develop a reliable highly distributed broadcasting protocol minimizing collisions and latency (especially in cases of public-safety related applications) without prior control messaging while considering different speeds, environments (urban and rural), and applications.

Many broadcasting algorithms have been introduced not matching the requirements of public safety applications as summarized in Sec. II. Therefore, we propose an application adaptive (multi-mode) headwaybased protocol for reliable broadcasting (particular for public -safety related messages) that is robust at different speeds and traffic volumes.

\section{Related Protocols}

We assume that the reader is familiar with the following acronyms [2]-[7]: Ready/Clear to Send (RTS/CTS), Contention window (CW), Short Interframe Space (SIFS), Distributed Coordination Function IFS (DIFS), Network Allocation Vector (NAV), and the Hidden node problem.

Based on the IEEE 802.11 standard [3], 1- "There is no MAC-level recovery on broadcast or multicast frames. As a result, the reliability of this traffic is reduced.", 2- "The RTS/CTS mechanism cannot be used for messages with broadcast and multicast immediate destination since there are multiple recipients for the RTS, and thus potentially multiple concurrent senders of the CTS in response." Existing VANET broadcasting protocols [4]-[15] just addressed 2 points: 1-How to deliver the broadcast to nodes within a single communication range with highest possible reliability, i.e. reliable protocols? and 2- How to deliver the broadcast to the entire network, i.e. dissemination protocols?

\subsection{Reliable Protocols}

Reliable protocols are managed by the source node only and are used with applications related to direct 
neighbors (e.g. public-safety applications). Broadcast reliability is increased through the following 3 approaches:

\subsubsection{Re-broadcasting of the same message for many times:}

The question is, how many times are considered practically enough? Xu [4] suggested that, rebroadcasting should be for a fixed number of times after sensing the channel as idle in each time. Yang [5] suggested re-broadcasting with a decreasing rate. Alshaer [6] proposed an adaptive algorithm where each node determines its own rebroadcast probability according to an estimate of vehicle density around it which is extracted from the periodic packets of routing management.

\subsubsection{Selective ACK:}

ACKing is the ultimate method of reliability, but with broadcasting we cannot let all receivers reply simultaneously. Tang [7] suggested unicasting the message to every node, one by one. Huang [8] suggested exchanging RTS/CTS with every node, then broadcasting the message once. Xie [9] proposed, on every broadcast, requesting ACK from only one receiver, on a round-robin style.

Changing transmission parameters: Balon [1] proposed decreasing collisions by changing the contention window size, based on an estimate of the current state of the network.

\subsection{Dissemination Protocols}

Dissemination protocols are managed by all nodes of the network, and are used with applications related to the entire network (e.g. traffic management). Here, the key design parameters are redundancy and dissemination speed. Researchers took 2 approaches to enhance the performance:

\subsubsection{Flooding:}

Flooding protocols are highly distributive, where it is each node's responsibility to determine whether it will re-broadcast the message or not. Ni [11] was the first to study flooding techniques in Ad-Hoc networks, and introduced the well-known "broadcast storm" problem. Then, he suggested that each node should only rebroadcast after comparing its location with the sender location and calculating the additional coverage it can provide. Heissenbüttel [12] proposed the same idea but, each node should introduces a back-off time that is shorter for greater additional areas.

\subsubsection{Single relay:}

We can mind single relay protocols as sequential ones, where the source node handles the responsibility of the broadcast to a next hop node. The question here is how to inform the next node of this new job. Zanella [13] proposed the Minimum Connected Dominating Set (MCDS), which is the minimum set of connected nodes that every other node in the network is one-hop connected with a node in this set. If the message was forwarded only by MCDS nodes, we would achieve the largest progress along the propagation line, while guaranteeing the coverage of all other network nodes, giving the theoretical optimal performance.

In the "Urban Multihop Broadcast Protocol (UMB)", Korkmaz [14] defined the term RTB/CTB (Ready/Clear to Broadcast), equivalent to the IEEE RTS/CTS, and suggested that the farthest node could be known by using black-burst, where its duration is longer for farther nodes. In the "The Smart Broadcasting Protocol (SB)" Fasolo [15] addressed the same idea but, using backoff time that is shorter for farther nodes. Reliable protocols care for all nodes randomly, but dissemination protocols care for the furthest node only.

\section{Proposed Protocol}

Giving more consideration to public-safety related applications, we propose a novel broadcasting protocol that is basically useful in emergency situations where the abnormal vehicle needs to open an instant communication channel with the vehicle(s) in the most dangerous situation.. Thus it is a case of unicast information packed in a broadcast protocol because there is not enough time for handshaking and moving to a service channel. But, it is worth emphasizing that it is still a broadcasting protocol in the sense that all surrounding vehicles within the communication range should receive and process the message while taking actions in their turn, especially if potentially probable to be affected by the danger. The question here is how to get ACK from the vehicle that is in the most dangerous situation.

In this section, we propose an application adaptive (multi-mode), headway-based protocol for reliable broadcasting (of public-safety related messages in particular) that is robust at different speeds and traffic volumes. We use the notation RTB/CTB as an equivalent to the IEEE RTS/CTS in broadcasting [3],[14]. Irrespective of the slightly increased overhead in case of short stream of data with the use of RTS/CTS, an appropriate node to reply with ACK (or CTS in case of long stream of data) is chosen. 
The proposed protocol involves the following assumptions and 4 proposed concepts/approaches, namely 1Reversing Order of Priority, 2- Headway-Based Segmentation, 3- Non-Uniform Segmentation based on naturalistic model of driver's reactions, and 4- Application Adaptive Multi-Mode schemes:

\subsection{Assumptions}

We assume that each vehicle involved in the protocol is at least equipped with: a high accuracy positioning device (GPS), one wireless transceiver $(5.9 \mathrm{GHz})$ and a speed sensor. The broadcasted message (RTB) contains the following: source node MAC address, the coordination of the source node, current traveling speed of the source node, the message propagation direction and broadcast mode (given later).

\subsection{Reversing the Order of Priority}

In almost all emergency situations (e.g. co-operative collision warning), the most threatened vehicle is the nearest one running behind the source vehicle. Hence, the first proposed approach is reversing the order of priority as shown in Fig.1. With this step, the protocol chooses the nearest node with a plain uniform distancebased segmentation algorithm. Though during communication between the source vehicle and the nearest following one, there could be collisions at far range nodes due to the hidden terminal problem, this choice gives the protocol an incomparable minimum latency. As a compensation for this type of collision, we recommend that, the ACK message should be the same as the broadcast one. Hence, we include an "ACK" field in the broadcast; which should be set in the ACK message.

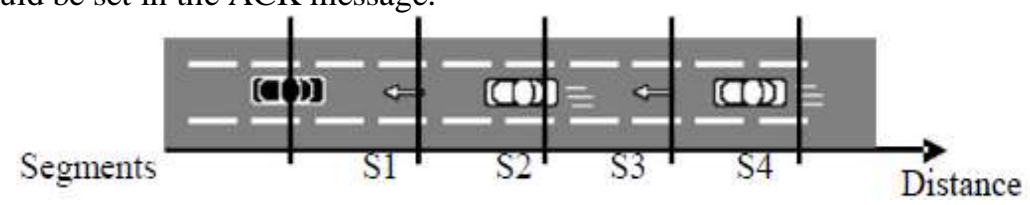

Fig. 1. Proper segmentation

\subsection{Headway Based Segmentation}

If vehicles are running at different speeds, distance-based segmentation logically fails in simulating the dangerous situation. Hence, the $2^{\text {nd }}$ proposed approach is to include the effect of speed through time headway based segmentation to assign segment numbers. The time headway or headway for short: is the time interval between two vehicles passing a point. Fig.2 shows a 3-lane highway with three following vehicles running at different speeds, $(30,60,120 \mathrm{Km} / \mathrm{h})$ with reference to distance (meter). Fig. 3 shows the same situation after calculating the headway for each vehicle to produce an imaginary calculated image. This image reveals that headway-based segmentation mimics dangerous situations better than distance -based one, as it puts the $120 \mathrm{Km} / \mathrm{h}$-vehicle in the $1^{\text {st }}$ priority, consistent with the intuitive analysis of the situation. So, the algorithm elects the nearest vehicle (in time) by a plain uniform headway-based segmentation method.

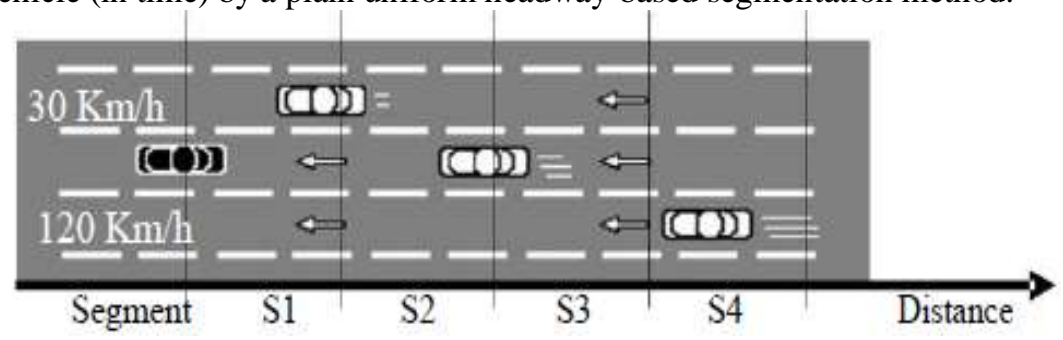

Fig.2. Distance-based segmentation

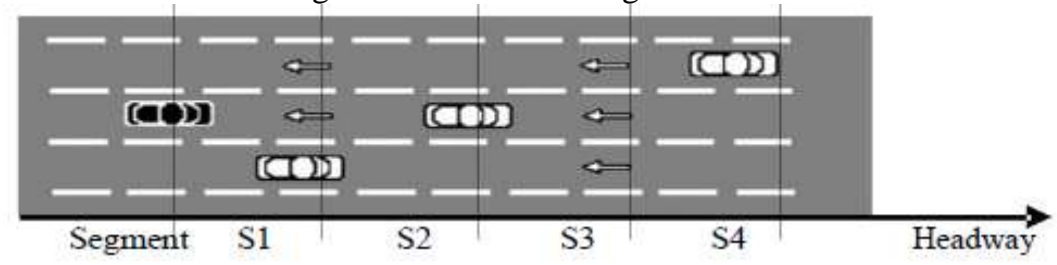

Fig.3. Time-based segmentation

\subsection{Non-Uniform Segmentation (Headway Model)}

We propose to let the width of each segment to be chosen according to the expected headway that drivers tend to leave apart. We adopt Semi-Poisson distribution headway model describing the average naturalistic headway that drivers tend to leave apart [16] as a basis for a non-uniform segmentation. Without loss of generality, assume only 2 vehicles in the transmission range of the source node. The headway between 
the source vehicle and the first one is $X_{1}$ sec, and the headway between those two vehicles is $X_{2}$ sec. Both $\mathrm{X}_{1}$ and $\mathrm{X}_{2}$ are random variables with a Semi-Poisson probability distribution function. We also assume that the highway is only one lane and both $\mathrm{CW} \min$ and $\mathrm{CW}_{\max }$ equals to one, i.e. there is no contention or random backoff. For studying the collision probability in one of the segments, we assume that the segment is in-between any arbitrary headways $l_{i}$ and $l_{f}$ sec. There will be a collision in the CTB message if there are more than one node in this segment. The probabilities of collision $\left(\mathrm{P}_{\mathrm{C}}\right)$, successful broadcast $\left(\mathrm{P}_{\mathrm{b}}\right)$, i.e. only one node in the segment, idle $\left(\mathrm{P}_{\mathrm{i}}\right)$, and prior nodes captured the broadcast phase $\left(\mathrm{P}_{\mathrm{o}}\right)$ are given as follows (with discretization):

$$
\begin{aligned}
& : P c=\sum_{x=l i}^{l f} \frac{P(X=x)}{P(l i<X<l f)} \times P(X<l f-x) \\
& : P b=\sum_{x=l i}^{l f} \frac{P(X=x)}{P(l i<X<l f)} \times P(X>l f-x) \\
& : P i=P(X>l f) \\
& : P o=P(X<l i)
\end{aligned}
$$

The objective of the non-uniform segmentation is to find the best points of segmentation within a single communication range $(10 \mathrm{sec})$ that results in linearly increasing $\mathrm{P}_{\mathrm{C}}$ 's with a minimum slope. There are two reasons behind minimizing the slope instead of the absolute minimum: 1 - Intuitively, vehicles in $1^{\text {st }}$ segments are more threatened than those in the last segments. Each vehicle is exposed to a danger that is inversely proportional to the time before collision, i.e. the headway time. 2- The other reason is a traffic concept that if there are no vehicles in the first segment, we can expect that the traffic is moderate or low, and let later segments be of a wider width.

\subsection{Application Adaptive (Multi-mode) Scheme}

Although the majority of VANET applications require message broadcasting, each application has its unique flavor and needs a special treatment. The main difference is which of the following vehicles should have the highest priority to respond first, either replying to the source vehicle or replaying to the following vehicles. Without loss of generality, we propose only 4 modes covering major applications:

\subsubsection{Mode 0- Basic Broadcasting}

The zero mode is the original basic mode, where broadcasting is omni-directional with no intended vehicle nor acknowledgment. This mode is still useful in VANET environment especially in case of the 'status message', where, as recommended by DSRC [17], every vehicle should broadcast its position, speed, direction of travel, and acceleration every $300 \mathrm{~ms}$, and this transmission is intended for all vehicles within 10-sec travel time.

3.5.2 Mode 1- Furthest Following Node: The intended vehicle in this mode is the physically furthest one following the transmitting node. This mode is suitable to work as a dissemination protocol for applications like "Traffic Information", and "Work Zone Warning". So, we recommend the regular distance-based protocols (e.g. The Smart Broadcasting Protocol [15]) to be used in this mode.

3.5.3 Mode 2 - Nearest Following Node (in time): The intended vehicle is the nearest one (in time) running behind the source vehicle. This mode is suitable to work as a reliable protocol for all public-safety related applications like "Cooperative Collision Warning" and "Stop Light Assistant". Our non-uniform headway-based protocol is superior in this mode.

3.5.4 Mode 3 - Furthest Leading Node: The intended vehicle is the furthest one leading the source vehicle as in Fig.4. This mode is suitable for emergency applications like "Approaching Emergency Vehicle" either it was an ambulance or a police car. In this case, the headway is identical to distance because the speed is constant (headway is measure with reference to source node speed). However, with headway-based protocols, we can implement a non-uniform segmentation based on headway studies. 


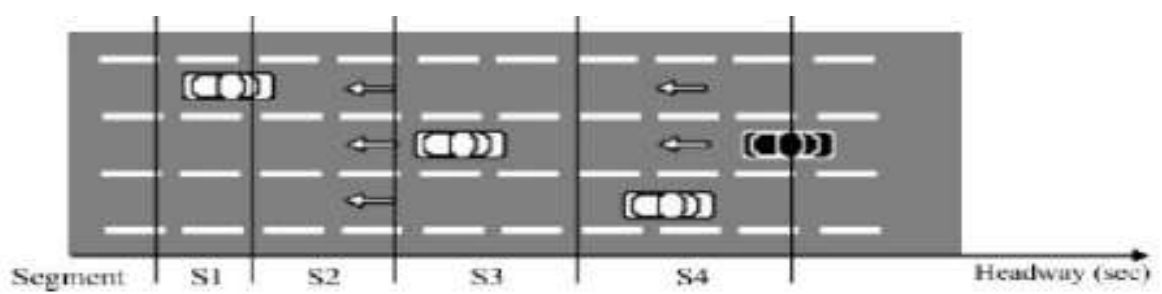

Fig. 4 Priority arrangement of mode 3.

\subsection{Proposed Protocol}

\subsubsection{Procedure of the source vehicle:}

In case of an OBU has a message to broadcast, the MAC layer of the system has to proceed with the following (Fig. 5):

1- It sends an RTB message including its MAC address, current location, mode of operation, .... etc..

2- It then waits for a valid CTB message within SIFS+N+1 time-slots (assuming N segments). If locked with a $\mathrm{CTB}$, then send the unencrypted broadcast with the intended receiver as that indicated in the CTB message. Otherwise (if not), repeat from Step-1 (as long as the application requires).

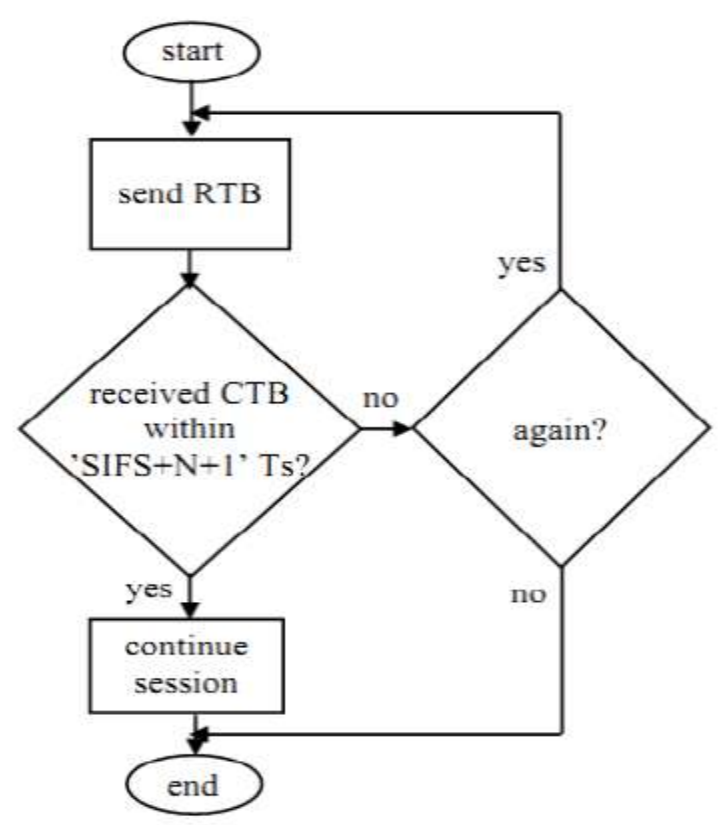

3.6.2 Procedure of other vehicles:

Fig.5. Actions of the source node.

Receiving of an RTB, other nodes proceed as follows (Fig. 6):

1- Set the NAV to be SIFS+N+2 time-slots so that no node will start a new session until the end of the current broadcast.

2- Check the broadcasting mode field.

3- Compare the geographical coordinates of the source node with their own and get its relative position. If the node is in the opposite driving direction or not in the message propagation direction, ignore it and go to end. However, if the node is in the message propagation direction, proceed to Step 4.

4 - Compute the headway in seconds (or distance in meter for mode 2), then determine its segment number.

5- If the segment number equals to $\mathrm{Si}$ where $(\mathrm{i}<=\mathrm{N})$ assuming ' $\mathrm{i}$ ' is the cell number, set the back-off counter $=$ i-1. Then, the node should wait for CTB message, if locked with a valid CTB then exit contention phase and listen for the coming broadcast. The node reaching 0 initiates the CTB including its MAC address and continues the session with the source node.

It should be noticed that in case of a lost source packet, the source sends again as long as the application requires. 


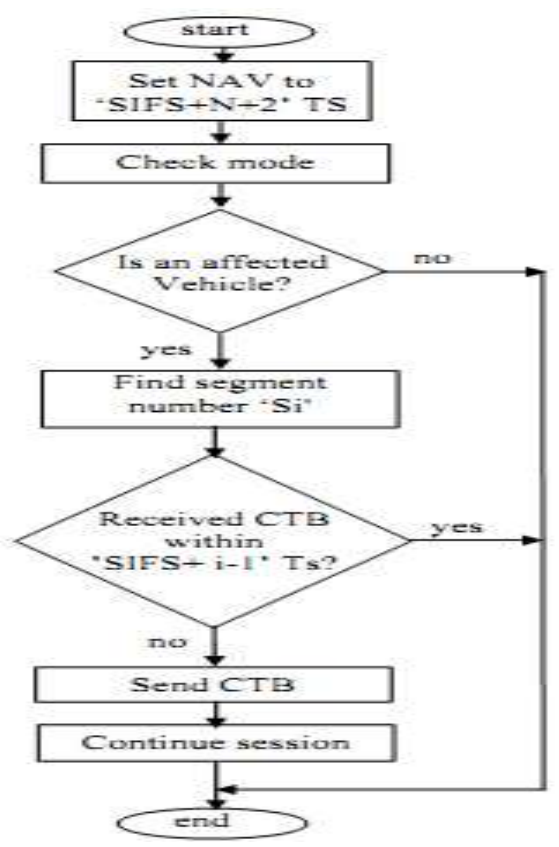

Fig.6. Actions of other nodes.

IV. Simulations and Results

Table 1 summarizes the assumptions taken during simulation, taken from the 802.11p [18] standard. Using these random variables, we conducted a simulation program for estimating the probability of collisions and the average latency within each segment. The latency is computed as typical based on [17]: contention starting time, success broadcasting time, collision time, and wait time, taking into account the MAC delay based on IEEE P802.11-REVma/D7.0 [18]. A 600 and $1600 \mathrm{veh} / \mathrm{h}$ traffic volume is considered in the headway-model.

Table 1. Simulation assumptions

\begin{tabular}{||c|c||c|c||}
\hline Time-Slot & $16 \mu \mathrm{s}$ & CTB & 14 bytes \\
\hline SIFS & $32 \mu \mathrm{s}$ & Messages & 512 bytes \\
\hline DIFS & $64 \mu \mathrm{s}$ & ACK & 512 bytes \\
\hline RTB & 20 bytes & Data rate & 3 Mbps \\
\hline
\end{tabular}

Using these random variables, a simulation program was conducted for estimating the probability of collisions and the average latency within each segment of the communication range $(10 \mathrm{sec})$. The width of each segment is taken according to Table 1 . The probability of collision is shown in Figure 7, while the average latency is shown in Fig. 8.

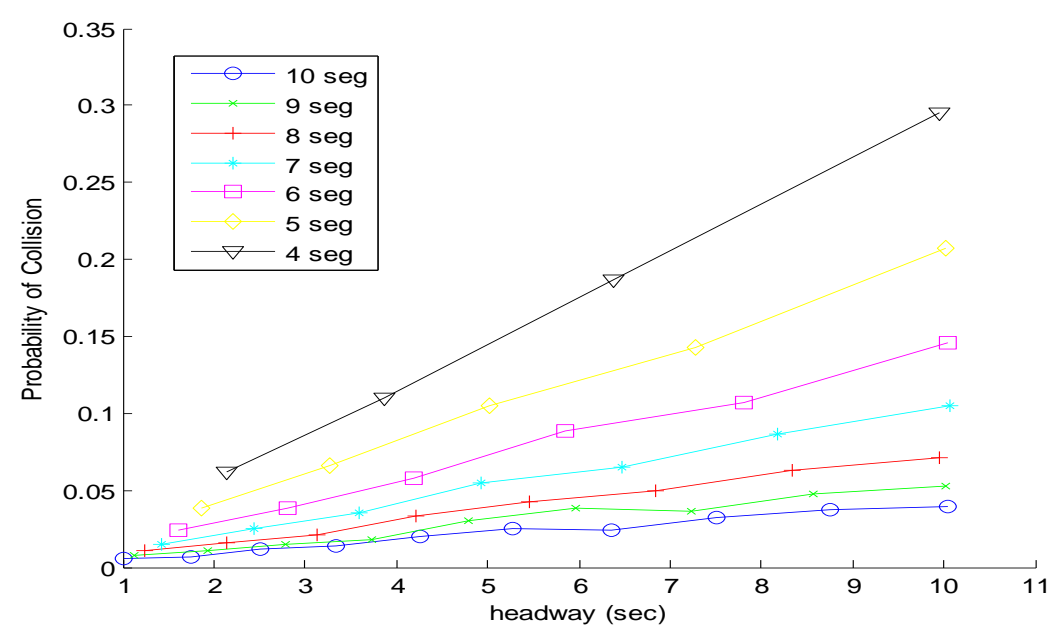

Fig. 7: Simulated calculation for $P C$ for best segmentation for $600 \mathrm{veh} / \mathrm{h}$. 
In Fig. 8, the curves are close to each other. The average latency associated with each segment reveals that the case of 7-seg gives the minimum latency (best performance) before over-segmentation begins to take place with 4 segments.

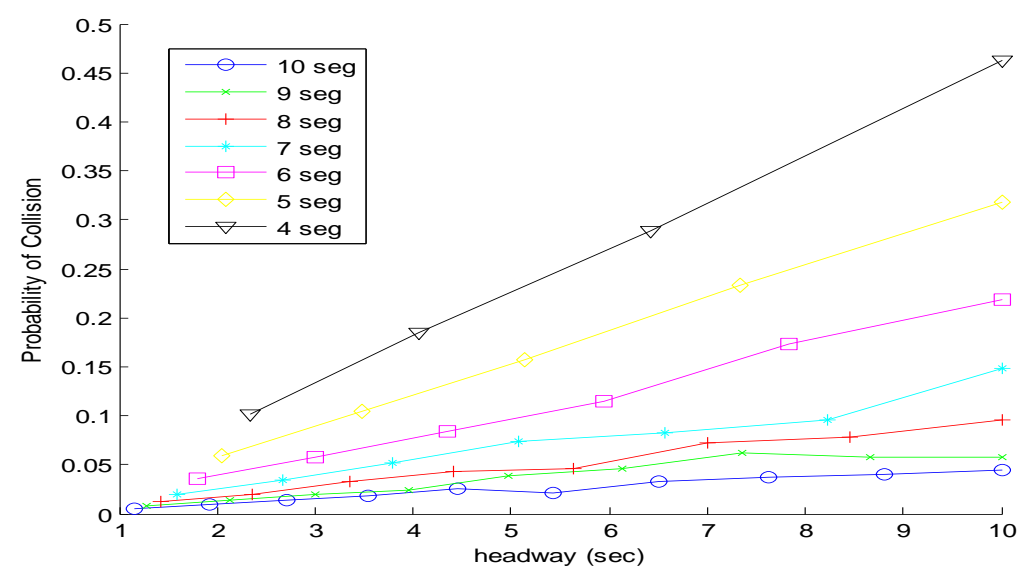

Fig. 8: Simulated calculation of latency at best segmentation for $600 \mathrm{veh} / \mathrm{h}$.

In Fig. 9, we have seen that for 1600 vehicles/h, the probability of collision is higher than the 500 vehicles/h. The latency of the segmentation for 1600 vehicles/h has given in Fig. 10.

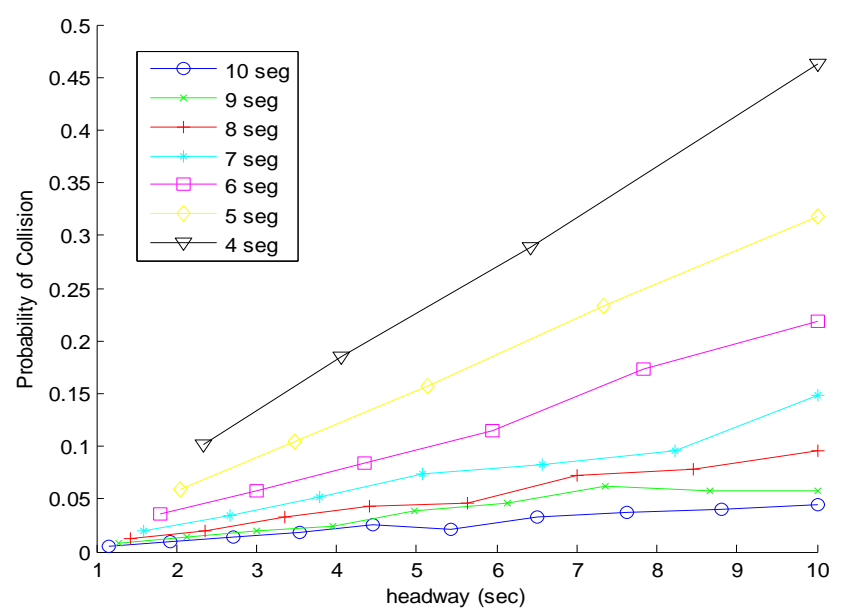

Fig. 9: Simulated calculation for $P C$ for best segmentation for $1600 \mathrm{veh} / \mathrm{h}$.

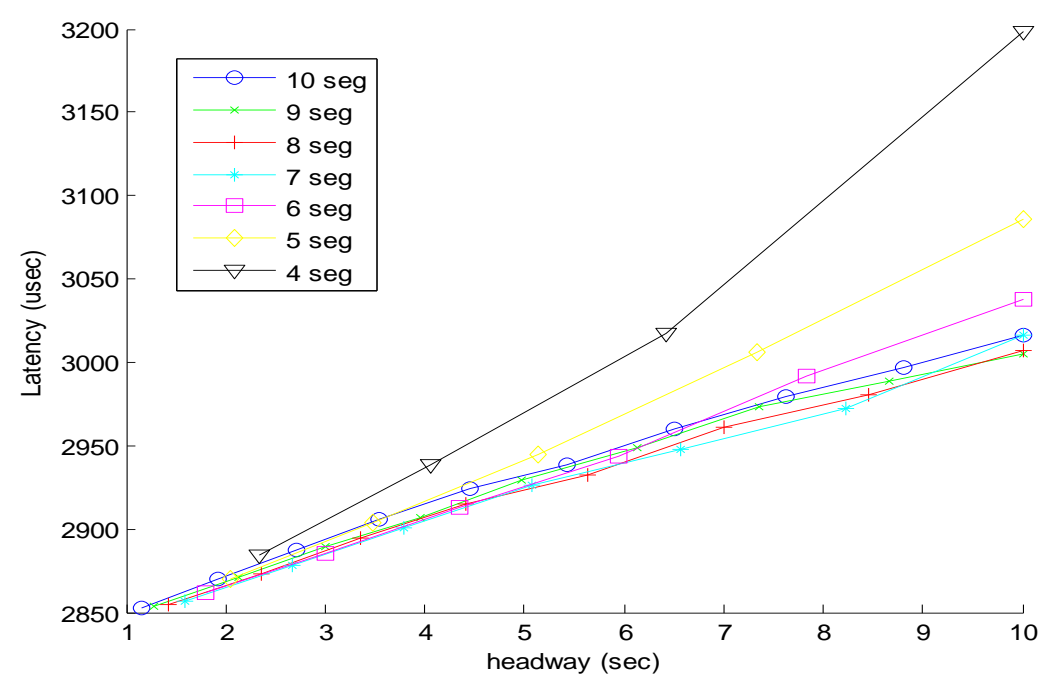

Fig. 10: Simulated calculation of latency at best segmentation for $1600 \mathrm{veh} / \mathrm{h}$. 


\section{Conclusion}

In this research, we introduced a novel broadcasting protocol in VANET environments with these new distinguishing features:

- The first protocol to use the concept of headway-based segmentation and to include effects of human behaviors in its design with the headway model.

- Non-uniform segmentation achieving a unique a minimum slope linearly increasing latency distribution.

- Unique robustness at different speeds and traffic volumes rooted to the headway robustness at different traffic volume variations. Superior minimum latency for public safety applications. Application adaptability with special multi-mode operations.

- Considered offering a solution to applications never discussed in literature, like "Approaching Emergency Vehicle". situations.

Further analysis and simulation will be conducted to accommodate more complicated highway

\section{References}

[1] "USA National Transportation Statistics 2007," Bureau of Transportation Statistics, USA, 2007.

[2] Y. M. Y. Hasan, M. M. I. Taha, S. Alshariff, and A. Alakshar, "Integrated intra-vehicle - VANET system for increasing the road safety," in Proc. Global Knowledge Forum NOOR-2008, Almadina, KSA, June 2008.

[3] IEEE P802.11p, “Amendment 3: wireless access in vehicular environments (WAVE)," Draft D0.26, January 2006

[4] Q. Xu, T. Mak, J. Ko, and R.Sengupta, "Vehicle-to-vehicle safety messaging in DSRC," in Proc. of the $1^{\text {st }}$ ACM Int. Workshop on Vehicular Ad Hoc Networks VANET'04, NY, USA, pp.19-28, 2004.

[5] X. Yang, L. Liu, N. H. Vaidya, and F. Zhao, "A vehicle-to-vehicle communication protocol for cooperative collision warning," in Proc. of the $1^{\text {st }}$ Int. Conf. on Networking and Services, pp.114-123, 2004.

[6] H. Alshaer and E. Horlait, "An optimized adaptive broadcast scheme for inter-vehicle communication." in Proc. IEEE $61^{\text {st }}$ Int. Vehicular Technology Conf. VTC'05, vol.5, pp.2840-2844, 2005.

[7] K. Tang and M. Gerla, "MAC reliable broadcast in ad hoc networks," in Proc. IEEE Military Communications Conference Communications for Network-Centric Operations: Creating the Information Force, vol.2, pp.1008-1013, vol.2, 2001.

[8] L. Huang, A. Arora, and T.H. Lai, "Reliable MAC layer multicast in IEEE 802.11 wireless networks," in Proc. of the IEEE Int. Conf. on Parallel Processing ICPP'02, Washington DC, USA, 2002.

[9] J. Xie, A. Das, S. Nandi, and A. K.Gupta, "Improving the reliability of IEEE 802.11 broadcast scheme for multicasting in mobile ad hoc networks," vol.1, pp.126-131, vol.1.2005.

[10] N. Balon, and J. Guo, "Increasing broadcast reliability in vehicular ad hoc networks," in Proc. of the $3^{\text {rd }}$ ACM Int. Workshop VANET'06, New York, USA, pp.104-105, 2006.

[11] S. Y. Ni, Y. C. Tseng, Y. S. Chen, and J. P. Sheu, "The broadcast storm problem in a mobile ad hoc network," in Proc. of the 5th ACM/IEEE int. conf. on Mobile computing and networking MobiCom'99, NY, USA, pp.151-162, 1999.

[12] M. Heissenbüttel, T. Braun, M. Wälchli, and T. Bernoulli, "Optimized stateless broadcasting in wireless multi-hop networks," in Proc. IEEE Infocom'06, Barcelona, April 2006.

[13] A. Zanella, G. Pierobon, and S. Merlin, "On the limiting performance of broadcast algorithms over unidimensional ad-hoc radio networks," in Proc. of WPMC'04, Abano Terme, Padova, Sep. 2004.

[14] G. Korkmaz, E. Ekici, F. Özgüner, and U. Özgüner, "Urban multi-hop broadcast protocol for inter-vehicle communication systems," in Proc. of the $1^{\text {st }}$ ACM Int. Workshop on Vehicular Ad Hoc Networks VANET'04, NY, USA, pp. 76-85, 2004.

[15] E. Fasolo, A Zanella, and M. Zorzi, "An effective broadcast scheme for alert message propagation in vehicular ad hoc networks," in Proc. of IEEE Int. Conf. on Communications ICC'06, vol.9, pp.3960-3965, 2006.

[16] T. Luttinen, "Statistical Analysis of Vehicle Time Headways," Teknillinen korkeakoulu, pp.155-172, 1996.

[17] "Dedicated Short Range Communications (DSRC) Home," http://www.leearmstrong.com/DSRC/DSRCHomeset.htm

[18] IEEE P802.11-REVmaTM/D7.0, "Wireless LAN medium access control (MAC) and physical layer (PHY) specifications", Rev. of 802.11-1999, June 2006.

[19] M. M. I. Taha and Y. M. Y. Hasan, "VANET-DSRC Protocol for Reliable Broadcasting of Life Safety Messages," in Proc. of the 7th IEEE Int. Symp. on Signal Processing \& Information Technology - ISSPIT'07, Cairo, Egypt, pp.105-110, Dec. 2007. 